\title{
Carnitine deficiency: clinical, morphological, and biochemical observations in a fatal case
}

\author{
ANDREW G. ENGEL, BETTY Q. BANKER, AND ROBERT M. EIBEN \\ From the Neuromuscular Research Laboratory and Department of Neurology, Mayo Clinic and \\ Mayo Foundation, Rochester, Minnesota, and the Departments of Pathology (Neuropathology), Pediatrics, \\ and Medicine (Neurology), Cleveland Metropolitan General Hospital and School of Medicine, \\ Case Western Reserve University, Cleveland, Ohio, USA
}

SUMMARY A fatal case of carnitine deficiency is described. The patient had intermittent metabolic acidosis, fluctuating hepatomegaly, and progressive muscle weakness since 22 months of age. One of two liver biopsies revealed lipid accumulation in the hepatocytes, and a muscle biopsy at age 5 years showed a lipid storage myopathy. Type 1 fibres were the most severely affected. Satellite and vascular endothelial cells also contained abnormal lipid deposits. Quantitative electron microscopy demonstrated an approximately 50 -fold increase in lipid material, and a twofold increase in mitochondria in myofibres. The muscle carnitine level was less than one-seventh of the lowest value encountered in 74 biopsies from non-weak or neuromuscular disease controls. The basic abnormality in this patient is assumed to be a defect in carnitine biosynthesis.

The biochemical basis of most muscle diseases is not known. Until recently, only the muscle glycogenoses conditioned by distinct enzyme deficiencies, and the myopathy associated with xanthine oxidase deficiency (Chalmers et al., 1969) could be considered as biochemically distinct. Since 1973 two metabolic defects of fatty acid catabolism, carnitine deficiency (Engel and Angelini, 1973), and carnitine palmityltransferase deficiency (DiMauro and DiMauro, 1973), have been discovered. The syndrome of muscle carnitine deficiency associated with weakness and triglyceride accumulation in muscle fibres has been referred to as type 1 lipid storage myopathy (Engel et al., 1974). Carnitine palmityltransferase deficiency is associated with attacks of myoglobinuria provoked by fasting or exertion, minimal or no lipid excess in the muscle fibres, and a normal muscle carnitine level (DiMauro and DiMauro, 1973).

Carnitine is essential for the transport of fatty acids into mitochondria where they can undergo beta-oxidation (Fritz, 1968). It is synthesised by liver, and then delivered to the tissues by the circulation

This work was supported in part by Grant NS-6277 (AGE) fron: the National Institutes of Health, and by a Research Center Grant (AGE) and a Research Grant (BQB) from the Muscular Dystrophy Association.

Address for reprint requests: Dr A. G. Engel, Department of Neurology Mayo Clinic, Rochester, MN 55901 USA.

Accepted 12 November 1976
(Lindstedt, 1967; Broquist and Tanphaichitr, 1973). As carnitine levels in skeletal muscle and heart greatly exceed the serum level (Cederblad and Lindstedt, 1972), uptake by these tissues probably depends on an active transport mechanism. Thus, hypothetically, muscle carnitine deficiency might be caused by impaired hepatic biosynthesis or by a transport defect (Engel et al., 1974).

Seven instances of carnitine deficiency have been reported to date (Engel and Angelini, 1973; Engel $e t$ al., 1974; Markesbery et al., 1974; Vandyke et al., 1975; Smyth et al., 1975; Karpati et al., 1975; Angelini et al., 1976; Boudin et al., 1976). In four of these the serum carnitine level was normal or only slightly decreased (Engel et al., 1974; Markesbery et al., 1974; Vandyke et al., 1975; Angelini et al., 1976), strongly suggesting that hepatic synthesis was intact, and that the transport mechanism was at fault. In one of these patients the liver carnitine concentration was also measured and was normal (Engel et al., 1974).

In two other patients both muscle and liver carnitine levels were depressed (Karpati et al., 1975; Boudin et al., 1976); in one of these the serum carnitine level was also measured, and was abnormally low (Karpati et al., 1975). Both these patients had intermittent episodes of hepatic enlargement and insufficiency, and intermittent attacks of metabolic acidosis. One of the two patients died, and at necropsy 
was found to have massive accumulation of triglycerides in skeletal muscle fibres, hepatocytes, and renal tubular epithelial cells, and a patchy increase of lipid material in the myocardial fibres (Boudin et al., 1976). Thus, in these two patients, a defect in hepatic synthesis had resulted in systemic carnitine deficiency. We here report another fatal case of carnitine deficiency. The patient suffered from intermittent attacks of metabolic acidosis, fluctuating hepatomegaly, and progressive muscular weakness since early childhood. A muscle biopsy in 1968, when the patient was nearly 5 years old, demonstrated a lipid storage myopathy. The patient died of his illness in 1971. Carnitine deficiency was established in 1973 by biochemical studies on an aliquot of the muscle biopsy specimen that had been preserved in liquid nitrogen.

\section{Case report}

The patient, an only child, was the product of a normal pregnancy and delivery. His birthweight was $3,600 \mathrm{~g}$, and subsequent growth and development were normal. He sat alone at $6 \frac{1}{2}$ months, walked alone by one year and walked very well by 14 months. He spoke single words at 8-9 months. He remained well until 22 months of age when he had his first of many bouts of 'laryngitis', which started with vomiting one evening. By the following afternoon he became very lethargic and was admitted to hospital in Geneva, Switzerland. Examination at that time showed dyspnoea and tachypnoea. The patient's voice was weak and his mouth remained open. Both lower extremities were slightly hypotonic. The deep tendon reflexes were normal. Examination of the spinal fluid gave normal results. He was in metabolic acidosis (blood pH: 7.22; serum bicarbonate: $8.1 \mathrm{mmol} / \mathrm{l}$; blood $\mathrm{pCO}_{2}: 2.8 \mathrm{kPa}$ ). On treatment with intravenous solutions, the metabolic acidosis disappeared over an eight day period. The patient was very weak when he was discharged from the hospital and had to learn to walk again. It was only from this time that the parents were aware of gait difficulties. The patient fell frequently, and displayed the Gower phenomenon when attempting to rise from a supine position. He tired easily when he walked; his voice was feeble, and he had ptosis of his eyelids which became more prominent later in the day.

At the age of 28 months, the patient was again hospitalised in Switzerland because of persistent weakness. At that time he weighed $14 \mathrm{~kg}$ and was $88.5 \mathrm{~cm}$ tall. His liver was enlarged to $6-7 \mathrm{~cm}$ below the right costal margin. He had a wide based stance, a waddling, uncertain gait, and a positive Gower sign. There was weakness of the facial muscles, and his mouth remained open. Muscle strength was markedly de- creased in all four extremities. The quadriceps muscles were of reduced bulk while the calf muscles appeared enlarged. The deep tendon reflexes were decreased in the lower extremities but were normal in the uppers. Sensory examination was normal. The serum lactic dehydrogenase level was $336 \mathrm{U} / 1$ (normal: 96-240). The serum alanine aminotransferase, aspartate aminotransferase, aldolase, and creatine phosphokinase activities were normal. Electromyographic studies, including motor nerve conduction studies in the lower limb, revealed no abnormalities.

At the age of 36 months the patient was again admitted to hospital for liver and muscle biopsies. The muscle biopsy was interpreted as normal. The liver sections showed perilobular steatosis. After this admission, the parents noted that the patient's voice and bite had become much weaker. During the next 15 to 18 months the child's weakness remained unchanged, and he had occasional upper respiratory infections. At $4 \frac{1}{2}$ years of age he again had a bout of vomiting and lapsed into metabolic acidosis. On readmission to the hospital, the blood $\mathrm{pH}$ was 7.22 , the serum bicarbonate $8.1 \mathrm{mmol} / \mathrm{l}$, and the blood $\mathrm{pCO}_{2}$ $2.5 \mathrm{kPa}$. The serum lactic acid level was increased to 5 $\mathrm{mmol} / \mathrm{l}$. The following day the patient's heart became weaker, and he was placed on a pacemaker and supported with positive respiration. He was comatose for two days and remained on the respirator for $3 \frac{1}{2}$ days. His general condition gradually improved over the next month. The liver and muscle biopsies were repeated at that time and were interpreted as normal.

In 1968, at the age of 4 years and 10 months (Figs. 1 and 2), the child was admitted to the Cleveland Metropolitan General Hospital. He now weighed $14.5 \mathrm{~kg}$, stood $103 \mathrm{~cm}$ tall, and his blood pressure was $90 / 60 \mathrm{mmHg}$. His mental status was normal. He showed substernal excavation, had ronchi over the right lung posteriorly, and his liver was palpable $1.5 \mathrm{~cm}$ below the right costal margin. The boy could not converge his eyes and had bilateral ptosis. He had moderate difficulty in swallowing and secretions frequently collected around his mouth. The facial muscles were weak and the tongue was small (Fig. 1). There was moderate weakness of the pharyngeal muscles, the gag reflex was hypoactive, and the voice hoarse and hypernasal. There was moderately severe weakness of the neck flexor and pectoral and pelvic girdle muscles. Strength in the abdominal and trunk muscles was fair. The hamstring, iliopsoas, and gluteal muscles were the weakest while the distal muscles of the extremities were of normal strength. The muscle mass was decreased, most conspicuously in the quadriceps. The gait was waddling and the Gower sign positive. The deep tendon reflexes were hypoactive except the ankle jerks which were normal. The rest of the neurological examination was normal. 


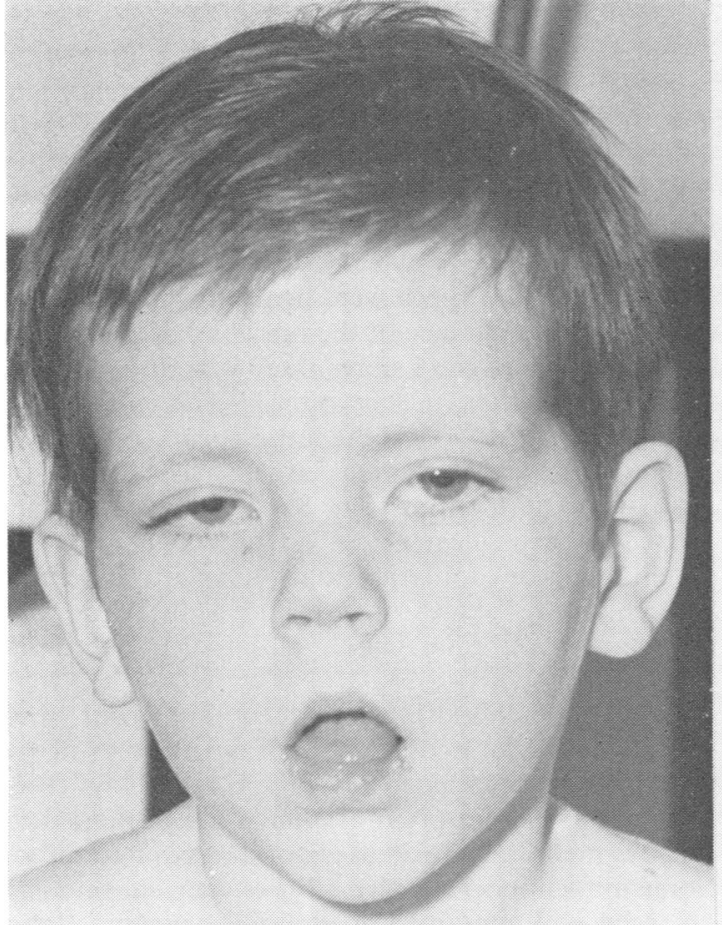

Figs. 1 and 2 Patient at the age of 4 years and 10 months.

Electromyographic studies demonstrated motor unit action potentials of very short duration in selected facial and extremity muscles. The incidence of polyphasic motor unit potentials was not increased, there was no abnormal irritability of the muscle fibres, and spontaneous electrical activity or myotonic discharges were not observed. The motor nerve conduction velocities were normal. Tests for a defect of neuromuscular transmission were negative.

A chest radiograph showed normal heart size and possible bronchiolitis. The electrocardiogram was normal. Routine examinations of blood and urine gave normal results except for the presence of a small number of monocytes with striking vacuoles in smears prepared from fresh capillary blood. The serum electrolytes and enzymes of muscle origin and the blood lactic acid level were normal. An adrenaline (epinephrine) tolerance test after an overnight fast resulted in a normal blood glucose response with minimal change of blood lactate. On the morning of this test the patient was in mild metabolic acidosis. However, he had vomited during the night and had not eaten well during the previous evening. After a 21 hour fast the patient developed ketonuria and his urinary $\mathrm{pH}$ fell to 5.7. However, he maintained his systemic acid-base equilibrium quite well with no change in blood $\mathrm{pH}$. A biopsy specimen was obtained from the left rectus femoris muscle in February 1968. The morphological and biochemical studies on this are described below.

The patient was followed as an outpatient in Cleveland until September 1968. When last seen, his clinical status was unchanged. However, the venous blood pH was 7.27 and the serum bicarbonate level $18.5 \mathrm{mmol} / \mathrm{l}$. His parents subsequently reported that the patient died a few weeks before his eighth birthday after a brief episode of respiratory distress, while on a holiday in France. Necropsy was not performed. No other member of the patient's family had suffered from a similar illness.

\section{HISTOPATHOLOGICAL STUDIES}

Specimens from the rectus femoris muscle were processed for ordinary light microscopy, light microscopic histochemistry, and for phase and electron microscopy. In addition, an aliquot was frozen in liquid nitrogen for subsequent biochemical investigations. Light microscopy In paraffin sections the muscle fibres ranged from 10 to $38 \mu \mathrm{m}$ in diameter. Many muscle fibres contained multiple, small, abnormal spaces that appeared empty. Central nuclei were not increased in number. Occasional regenerating but no 
necrotic fibres were observed. The connective tissue elements were normal.

Histochemistry The histochemical analysis was based on previously described methods (Dubowitz et al., 1973). In fresh frozen sections the abnormal spaces in the muscle fibres stained positively with lipid stains (Scharlach R. oil red 0, Sudan black B, Nile blue A) (Fig. 3). The lipid bodies separated the myofibrils and distended the intermyofibrillar space. In many fibres they were especially prominent under the sarcolemma, and conferred an irregularly undulating appearance on the fibre boundary. The abnormal deposits were largest and most abundant in type 1 fibres; type 2 fibres contained less lipid. Intermediate fibres contained intermediate amounts of lipid. As judged by the myofibrillar ATPase and by oxidative enzyme reactions, the normal checkerboard distribution of the histochemical fibre types was preserved. Because of the relative abundance of the abnormal spaces in the different fibre types, one could identify them even in H\&E and trichromatically stained sections. Type 1 fibres varied from 10 to 52 $\mu \mathrm{m}$ in diameter, while type 2 fibres ranged from 28 to $52 \mu \mathrm{m}$ in diameter. Subsarcolemmal and intermyofibrillar oxidative enzyme activity was increased in numerous fibres, and especially in type 1 fibres.

Phase microscopy Phase microscopic examination of glutaraldehyde-osmium fixed, epon embedded sections again revealed the abnormal spaces in the fibres, and one could again distinguish between the fibre types according to the abundance of the abnormal spaces. Of 107 fibres analysed by phase microscopy, $60.5 \%$ were judged as type 1 or inter- mediate, and $33.5 \%$ as type 2 . In these sections, capillaries appeared to be more numerous in relation to the type 1 fibres than other fibres. To quantitate this observation, the capillaries were counted in relation to 157 fibres. The capillary muscle fibre ratio was 1.84 for the type 1 fibres, 0.92 for the type 2 fibres, and 1.20 for the intermediate fibres.

Electron microscopy As in the phase microscopic studies, the fibre types were again distinguished by the relative abundance of the lipid bodies harboured by them. In the type 1 fibres there were myriad lipid deposits between the myofibrils and under the sarcolemma (Fig. 4). In the latter location the lipid bodies coalesced, forming larger masses with irregularly scalloped borders. Such masses often occurred in perinuclear zones, or in areas adjacent to satellite cells or capillaries. In the most affected fibres mitochondria frequently surrounded the borders of the intermyofibrillar and subsarcolemmal lipid deposits.

Most mitochondria contained numerous and elaborate cristae. In one-eighth of type 1 fibres many mitochondria contained crystalloid inclusions (Fig. $5 \mathrm{a}, \mathrm{b})$. These were often asymmetrically situated in the organelle, giving it a pistol-like configuration. Some mitochondria in type 1 fibres were enormously enlarged and contained crystalloid inclusions, lipid 2 을 droplets, or both (Fig. 5c). In many abnormal mito묵 chondria there were small abnormal spaces, often $\delta$ limited by double membrane. These spaces, which were either empty or contained degraded material, probably arose from invaginations of the mitochondrial membranes (Fig. 5a, b). Occasionally the mito-

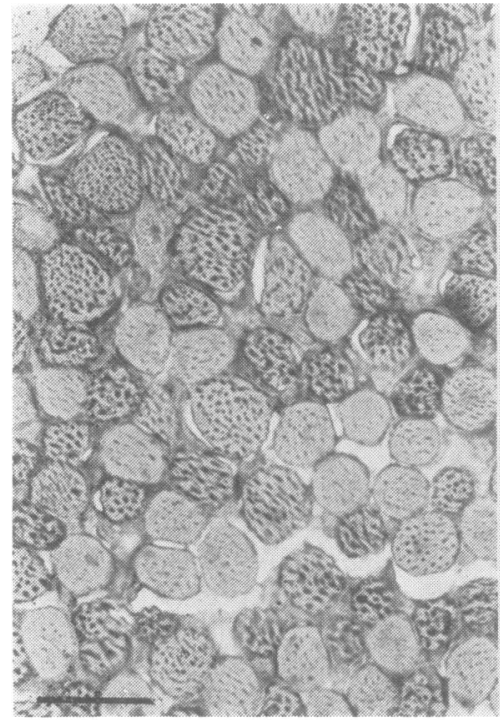

(a)

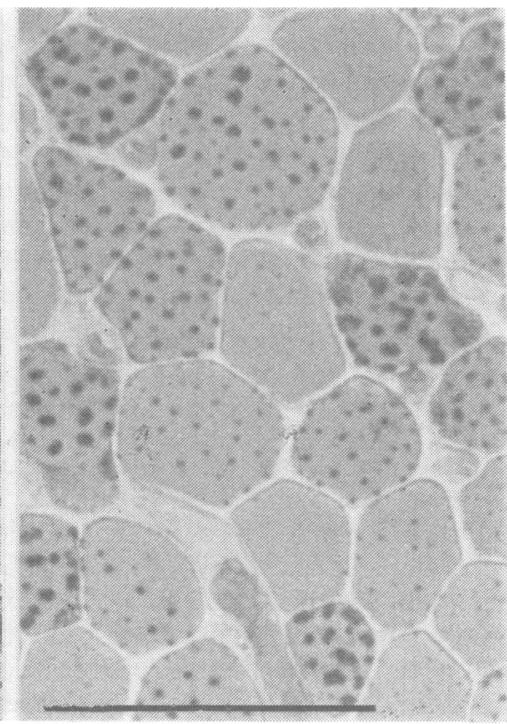

(b)
Fig. 3 Frozen sections stained with oil red $O$ (a) and Nile blue $A$ (b). According to amount of lipid accumulating in the fibres they can be divided into three types. Markers: $50 \mu \mathrm{m}$. 


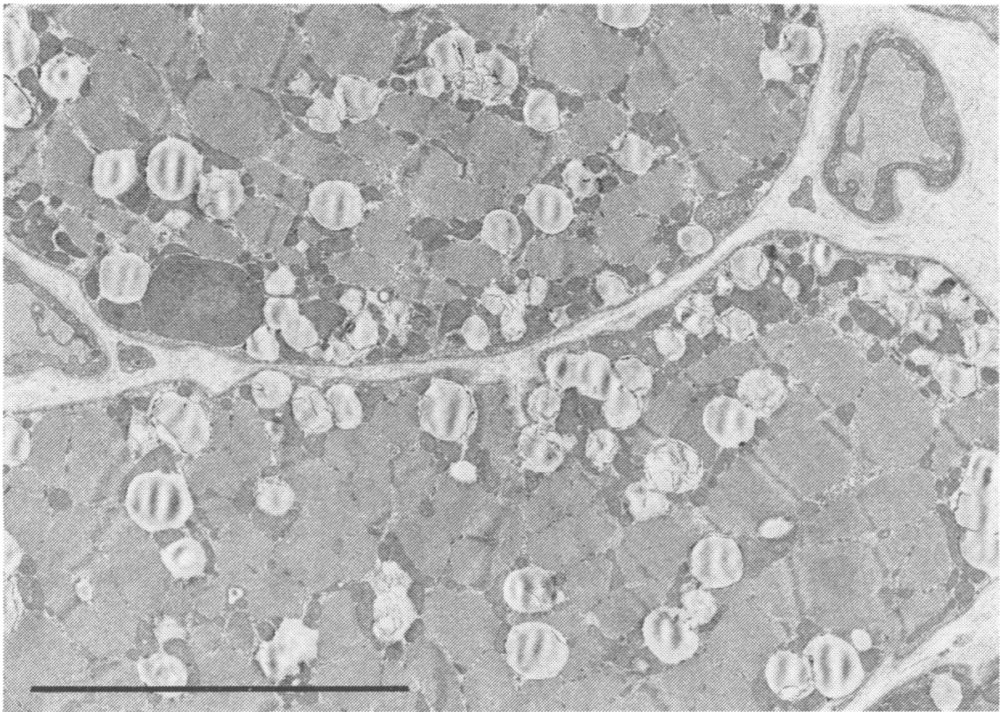

Fig. 4 Two muscle fibres contain rumerous lipid droplets surrounded by mitochondria. Some of the adjacent droplets are coalescing. Marker: $10 \mu \mathrm{m}$.

chondria harboured small lipid droplets or numerous electron dense granules.

In the less severely affected type 2 fibres the lipid bodies were still more conspicuous than in normal muscle. Here, however, the lipid deposits were smaller and less numerous than in the type 1 fibres, did not bulge the fibre borders and were not surrounded by mitochondria.

Satellite cells were unusually prominent in association with type 1 fibres. Of the 91 satellite cells encountered, $84(92.3 \%)$ were associated with type 1 or intermediate fibres, which, in turn, accounted for only $69.5 \%$ of the 308 fibres observed in the electron microscope. It was of special interest that 14 of the 91 satellite cells contained lipid droplets. Finally, lipid droplets were also conspicuous in the cytoplasm of pericytes, in the endothelial cells of capillaries and venules and in occasional fibroblasts (Fig. 6).

Morphometric analysis of electron micrographs Randomly selected, transversely oriented blocks were sectioned. All muscle fibres and fibre regions encountered on the grids were photographed once in a single plane of sectioning. Thirty-four muscle fibres were photographed so that large areas of the fibres were represented in the micrographs. The 74 micrographs thus obtained were then montaged to reconstruct the fibres as seen in the electron microscope. In addition, 32 micrographs were taken of single regions in 32 fibres. As the area of those fibres appearing on the grid was relatively small, montages could not be prepared. The two sets of micrographs were separately analysed. For each set, the total fibre areas (excluding the nuclei), and the mitochondrial and lipid fractions of the fibre areas, were measured with a Hewlett
Packard 9864A digitiser serving as a peripheral to a 9821A programmable calculator. The results were compared with morphometric data obtained in the Mayo muscle laboratory on transversely sectioned muscle fibres in four non-weak children (one month to 12.6 years of age) (Jerusalem et al., 1975). The results are shown in Table 1 . Inspection of the table indicates that (1) in the two sets of micrographs from the patient the findings were essentially identical; (2) in the patient, lipid bodies and mitochondria each occupied about $10 \%$ of the fibre volume; (3) the lipid content in the patient's fibres was increased approximately 50 -fold, and the mitochondrial volume was increased about twofold over the control values.

To see whether a correlation existed between the lipid and mitochondrial contents of the fibres, the mitochondrial fractions in the montaged fibres were plotted against the lipid fractions in these fibres. Regression analysis indicated a highly significant linear correlation between the two variables (correlation coefficient: $0.703, \mathrm{P}<0.001, \mathrm{~N}=34$ ).

\section{Biochemical studies}

The carnitine content of the patient's muscle was determined by the method of Marquis and Fritz (1964). The result was compared with carnitine levels in muscle specimens from 21 non-weak and 53 neuromuscular disease controls. In the control subjects, the assay of Marquis and Fritz or of Cederblad and Lindstedt (1972) was used. In 10 of the control subjects paired comparison of the two methods gave essentially identical results. The results are shown in Table 2. 

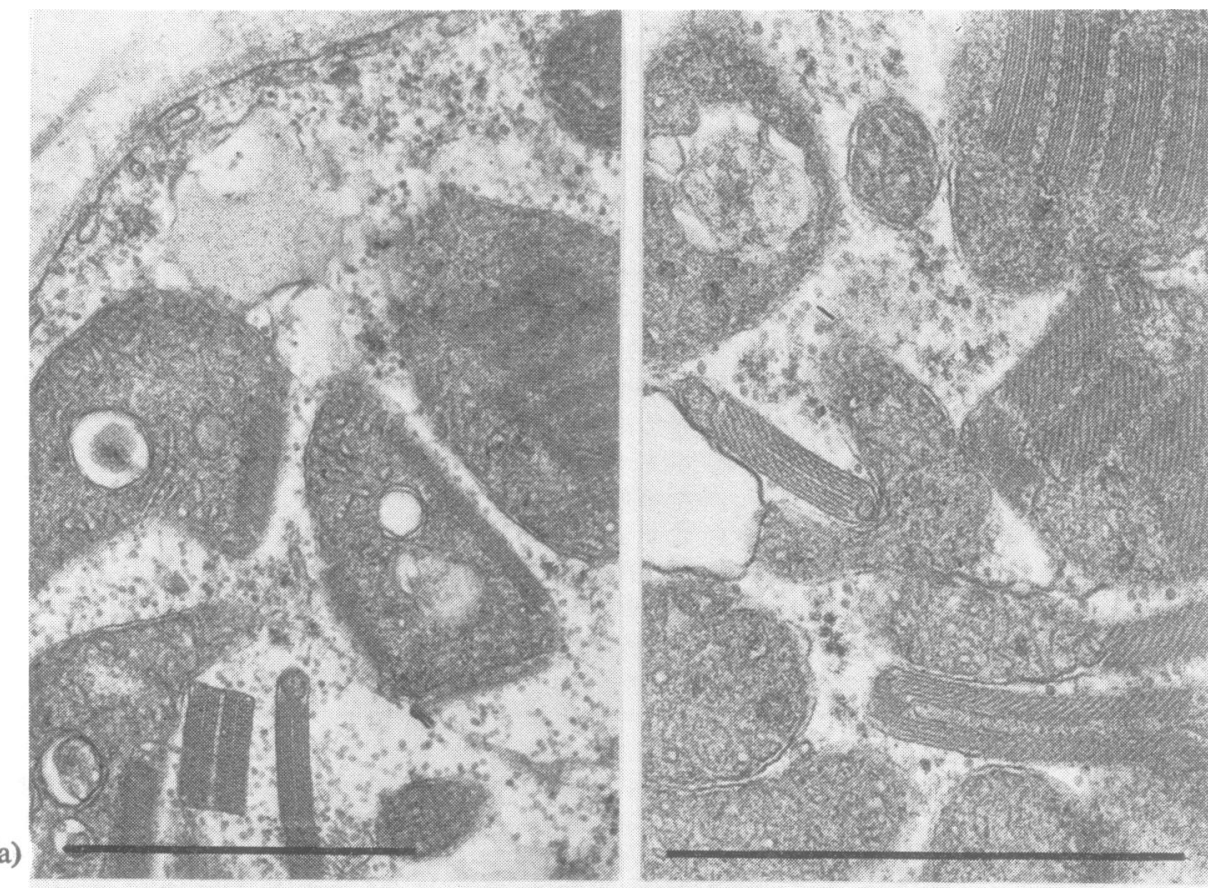

(b)

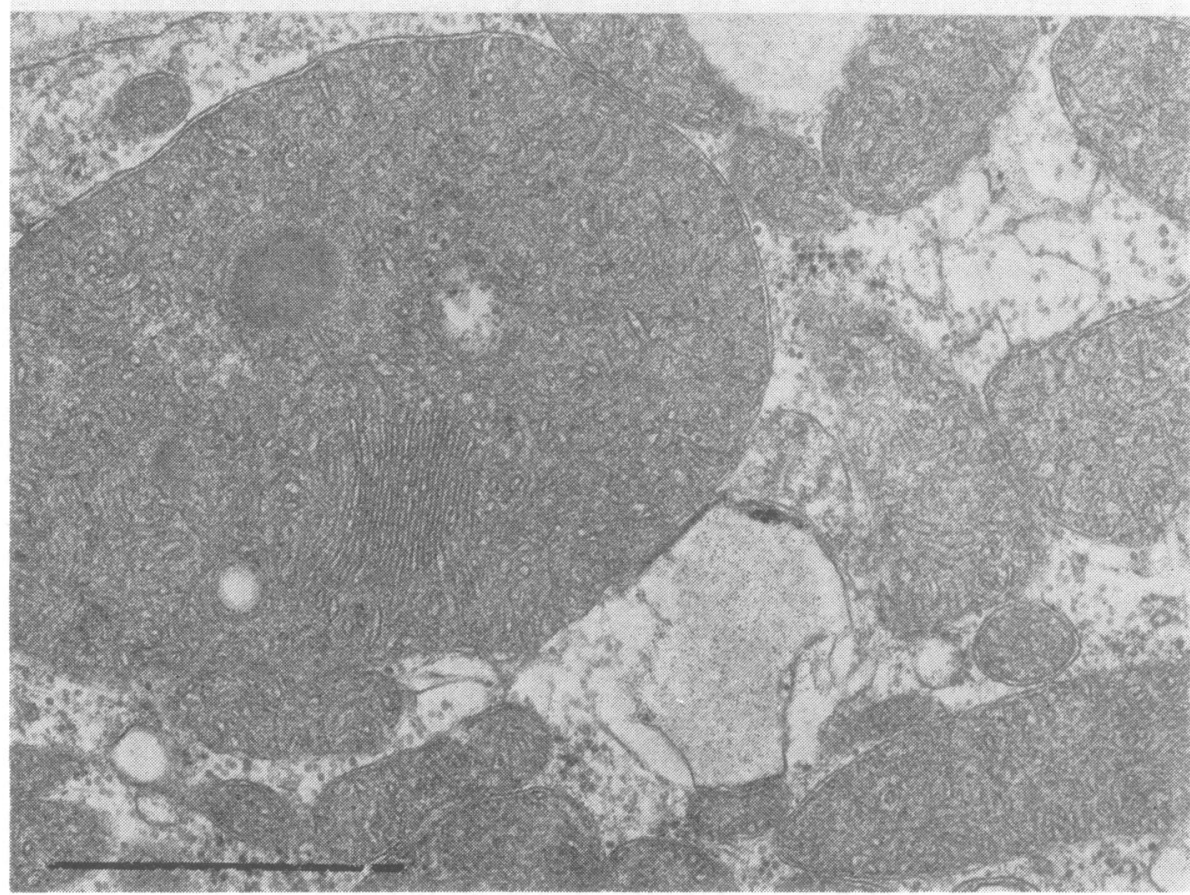

(c)

Fig. 5 Abnormal mitochondria containing crystalloid inclusions ( $a$ and $b$ ) or homogeneous material of medium electron density $(c)$, and cytoplasmic invaginations ( $a$ and $b)$. Mitochondrion at left of panel C is enormously enlarged. Markers: $1 \mu \mathrm{m}$. 


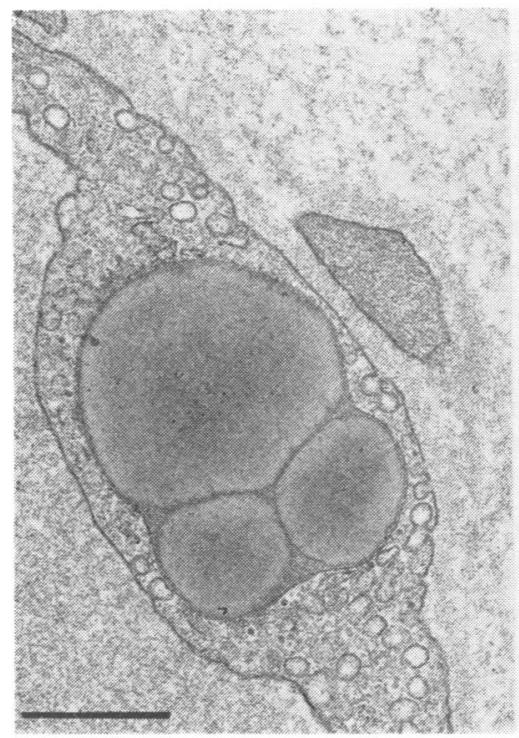

(a)

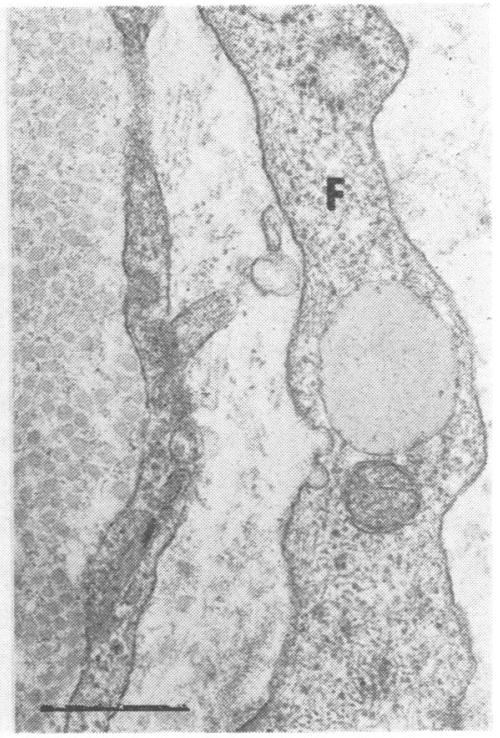

(b)
Fig. 6 Lipid bodies within cytoplasm of capillary endothelial cell (a) and fibroblast (b). F: Fibroblast process. Markers: $0.5 \mu \mathrm{m}$.
Table 1 Morphometric analysis of electron micrographs*

\begin{tabular}{lrr}
\hline & $\begin{array}{l}\text { Lipid content } \\
\%\end{array}$ & $\begin{array}{l}\text { Mitochondrial } \\
\text { content } \\
\%\end{array}$ \\
\hline 4 Control children & $0.19 \pm 0.08$ & $5.02 \pm 0.37$ \\
$\begin{array}{l}\text { (23 fibres) } \\
\text { Patient }\end{array}$ & $11.34 \pm 1.56$ & $10.44 \pm 1.17$ \\
34 montaged fibres & $9.42 \pm 1.61$ & $9.68 \pm 1.26$ \\
\hline 2 fibre regions & \\
\hline
\end{tabular}

*Values indicate Mean $\pm \mathrm{SE}$. The analyses were on transversely sectioned muscle fibres.

Table 2 Muscle carnitine levels

\begin{tabular}{ll}
\hline Source & $\begin{array}{l}\text { Carnitine } \\
\text { nmol/mg non-collagen } \\
\text { protein }\end{array}$ \\
\hline Patient & 0.94 \\
Non-weak controls & $14.57 \pm 0.93$ \\
Mean \pm SE & 7.96 to 22.86 \\
Range & 21 \\
N & $13.79 \pm 0.63$ \\
Neuromuscular disease controls & 7.43 to 27.77 \\
All cases & 53 \\
Mean \pm SE & \\
$\mathbf{N}$ & $14.42 \pm 0.70$ \\
Disease controls excluding Duchenne & 8.32 to 27.77 \\
dystrophy & 42 \\
Mean \pm SE & $11.38 \pm 1.19$ \\
Range & 7.43 to 19.17 \\
$\mathbf{N}$ & 11 \\
Duchenne dystrophy & \\
Mean \pm SE & \\
Range & \\
N & \\
\hline
\end{tabular}

Inspection of the table shows that the patient's muscle carnitine level was less than one-seventh of the lowest value encountered in either of the two control groups. Since some of the lowest neuromuscular disease control values occurred in Duchenne type muscular dystrophy, the table also compares the Duchenne patients with the remaining neuromuscular disease controls. A barely significant difference $(P=0.049)$ exists between the Duchenne and other disease controls. Figure 7 shows the distribution of carnitine levels according to diagnosis in $\mathbf{4 5}$ disease controls, as well as in the non-weak subjects, and in the patient.

\section{Discussion}

The clinical features of the patient strongly suggest that he had suffered from systemic carnitine deficiency due to a defect in hepatic carnitine synthesis. As in the other two patients with systemic carnitine deficiency (Karpati, et al., 1975; Boudin et al., 1976), he had had fluctuating hepatomegaly, intermittent attacks of metabolic acidosis, and his muscle weakness had begun in early childhood. The patient's liver, when it was maximally enlarged, showed excessive lipid deposits in the hepatocytes. Seven months later, however, another liver biopsy was interpreted as normal. This does not exclude the diagnosis for in the patient with systemic carnitine deficiency studied by Karpati and his co-workers (1975), a liver biopsy also failed to show lipid excess at a time when the patient was not critically ill. 


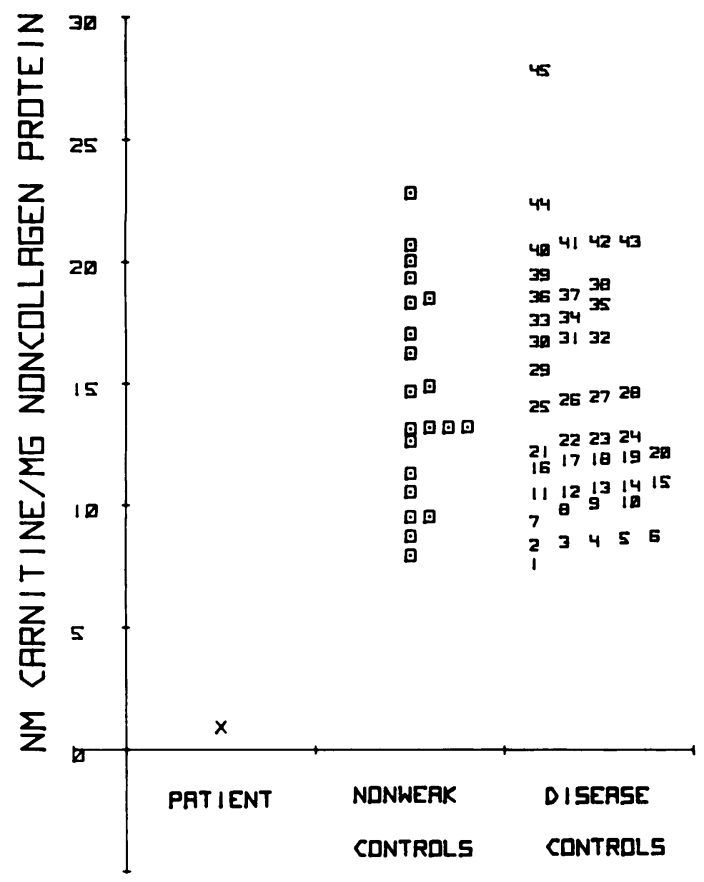

Fig. 7 Muscle carnitine levels in patient and controls. Neuromuscular disease controls represented by number as follows: Duchenne dystrophy 1, 2, 15 (eight additional Duchenne cases are not represented in the graph but are included in Table 2); motor neurone disease 3, 11; limbgirdle dystrophy 4, 8, 24, 41; Becker dystrophy 5; adult polymyositis $6,10,13,17,27$; mitochondria-lipidglycogen disease 7, 22, 25; nemaline myopathy 9; lipid storage myopathy not due to carnitine deficiency 12, 30; mitochondrial myopathy not involving ocular muscles 14, 29, 34, 45; ocular-cranial-limb muscle dystrophy 16, 18, 37, 40, 44; diabetic neuropathy 19, 28, 38, 42; central core disease 20; malabsorption syndrome 21; myotonic dystrophy 23, 32; carnitine palmityltransferase deficiency 26; childhood dermatomyositis 31,36 ; thyrotoxic myopathy 33; steroid myopathy 35; adult acid maltase deficiency 39; myxoedema myopathy 43.

The patient's metabolic acidosis could at least par tially be attributed to lactic acidosis, a feature that was also observed in the two patients with systemic carnitine deficiency studied by Karpati and coworkers (1975), and by Boudin et al. (1976). (Lactic acidosis was also reported in the patient described by Smyth et al. (1975) but neither plasma nor liver carnitine levels were given in this patient). The lactic acidosis might be a consequence of (1) the accelerated glycolysis when fatty acids are unavailable as an energy source, and (2) impaired use of lactate by the liver for gluconeogenesis, which itself is an energyrequiring process (Lehninger, 1970). An additional factor that might have contributed to the acidosis could have been accelerated omega-oxidation of fatty acids giving rise to serum and urinary dicarboxylic acids, as was demonstrated in Karpati's patient with systemic carnitine deficiency. The omega oxidation is extramitochondrial and not carnitine dependent, and is believed to occur in the endoplasmic reticulum of liver cells. A third possible mechanism of ketoacidosis in systemic carnitine deficiency would be the accelerated oxidation of ketogenic branched-chain amino acids, namely leucine, isoleucine, and valine. These amino acids are preferentially catabolised by skeletal muscle (Buse et al., 1972; Odessey et al., 1974; Goldstein and Newsholme, 1976). The initial step in their metabolism is transfer of their amino group to pyruvic acid. The alanine thus formed is released from muscle, and after being taken up by liver, is reconverted to pyruvic acid (Felig, 1975). If fatty acids are stored in muscle and are unavailable as a source of energy, increased utilisation of carbohydrate, yielding pyruvic and lactic acids, as well as of ketogenic branched-chain amino acids is likely to occur. While a number of factors can account for the metabolic acidosis in systemic carnitine deficiency, the reason for its intermittent character, and for the intermittent liver enlargement, is not known a present.

There were no signs of cardiac involvement in ours case. This does not exclude a subclinical cardiomyo $\rightarrow$ pathy, for in the patient investigated by Boudin et al. $\Omega$ (1976) there were lipid deposits in the myocardiate fibres without clinical cardiac involvement. However clinical cardiomyopathy has been observed in onecase of carnitine deficiency (Vandyke et al., 1975). Excessive accumulation of lipid droplets in Schwann cells and a peripheral neuropathy have also been observed in one patient with carnitine deficiency (Markesbery et al., 1974), but not in others. Variable involvement of different tissues could occur whether the carnitine deficiency is conditioned by a transport defect or by impaired hepatic synthesis, depending on the carnitine demands of the tissues, and on the varying capabilities of the cells in different tissues to take up carnitine against a concentration gradient. Variations in the clinical expressivity of inborn errors of metabolism are readily accounted for in terms of genetic heterogeneity (Sutton and Wagner, 1975). Nonetheless, it is clear that muscle weakness and lipid excess are common features of all cases of carnitine deficiency.

The morphological alterations in the patient's muscle fibres were essentially like those in other patients with carnitine deficiency. Type 1 fibres contained the most lipid deposits and, apart from the mitochondria, no other organelles responded pathologically. The presence of lipid bodies in satellite cells and in vascular endothelial cells was of special 
interest, for this has not been noted previously in carnitine deficiency.

The predilection of satellite cells to type 1 and intermediate fibres was readily appreciated. These observations were enhanced by the disease markerthe accumulation of lipid and mitochondria in the type 1 and inter mediate muscle fibres. In various other diseases of muscle it is difficult to differentiate the three fibre types without such an ultrastructural marker. For this reason, data regarding such satellite cell-fibre type colrelation are lacking. Without these data, it is difficult to understand the significance of the selectivity of the satellite cell to the type 1 and intermediate muscle fibres in carnitine deficiency. The most logical explanation would be that the increase in number of satellite cells occurs in association with the abnormal muscle fibres. It follows that the satellite cells represent a non-specific reaction to an alteration within these specific muscle fibres.

Morphometric study of the electron microgiaphs gave evidence for an approximately 50 -fold increase in lipid and a twofold increase in the mitochondrial volume in the fibres analysed. Further, there was a linear correlation between the lipid and mitochondrial increases in the individual fibres. The mitochondrial increase was interpreted as a compensatory reaction. In the presence of reduced carnitine levels in the muscle fibres, the rate of fatty acid oxidation by each mitochondrion is likely to be depressed. An increase in the mitochondrial volume might result in greater rate of fatty acid oxidation by the entire fibre.

The significance of the crystalloid inclusions in some of the mitochondria is not clear. They have been found in other patients with carnitine deficiency (Karpati et al., 1975; Boudin et al., 1976), and also occur commonly in other myopathies associated with large or over-abundant mitochondria (Dubowitz et al., 1973). Clearly they represent a nonspecific response to diverse metabolic abnormalities.

The fact that an increasing number of patients have been diagnosed as suffering from carnitine deficiency since the initial description of the syndrome in 1973 suggests that the disease is not uncommon. While not all cases of lipid storage myopathy are due to carnitine deficiency (Gulotta et al., 1974; Jerusalem et al., 1975), recognition of the disease by the appropriate histological and biochemical tests is important because it is potentially fatal, and because therapy with carnitine (Engel et al., 1974; Angelini et al., 1976; Karpati et al., 1975), or with prednisone (Engel and Angelini, 1973; Vandyke et al., 1975) has been of benefit in several instances.

We are grateful to Professor Fred Bamatter of Geneva, Switzerland, for providing us with detailed summaries of the patient's hospitalisation in Switzer- land, and to Dr Robert Schwartz, Professor of Pediatrics, Brown University, Providence, Rhode Island, for allowing us to study his patient.

\section{References}

Angelini C., Lücke, S., and Cantarutti, F. (1976). Carnitine deficiency of skeletal muscle: Report of a treated case. Neurology (Minneapolis), 25, 633-637.

Boudin, B., Mikol, J., Guillard, A., and Engel, A. G. (1976). Fatal systemic carnitine deficiency with lipid storage in skeletal muscle, heart, liver and kidney. Journal of the Neurological Sciences, 30, 313-325.

Broquist, H. P., and Tanphaichitr, V. (1973). Site of carnitine (carn) biosynthesis in the rat. Federation Proceedings, 32, 884.

Buse, M. G., Biggers, J. F., Fredereici, K. H., and Buse, J. F. (1972). Oxidation of branched chain amino acids by isolated hearts and diaphragms of the rat. Journal of Biological Chemistry, 249, 8085-8096.

Cederblad, G., and Lindstedt, S. (1972). A method for the determination of carnitine in the picomole range. Clinica Chimica Acta, 37, 235-243.

Chalmers, R. A., Johnson, M., Pallis, C., and Watts, R. W. E. (1969). Xanthinuria with myopathy. Quarterly Journal of Medicine, New Series, 38, 493-512.

DiMauro, S., and DiMauro, P. M. M. (1973). Muscle carnitine palmityltransferase deficiency and myoglobinuria. Science, 182, 929-931.

Dubowitz, V., Brooke, M. H., and Neville, H. E. (1973). Muscle Biopsy: A Modern Approach. W. B. Saunders: Philadelphia.

Engel, A. G., and Angelini, C. (1973). Carnitine deficiency of human muscle with associated lipid storage myopathy: A new syndrome. Science, 179, 899-902.

Engel, A. G., Angelini, C., and Nelson, R. (1974). Identification of carnitine deficiency as a cause of human lipid storage myopathy. In Exploratory Concepts in Muscular Dystrophy II, pp. 601-617. Edited by A. T. Milhorat. Excerpta Medica: International Congress Series No. 333, Amsterdam.

Felig, P. (1975). Amino acid metabolism in man. Annual Review of Biochemistry, 44, 933-955.

Fritz, I. B. (1968). The metabolic consequences of the effect of carnitine on long chain fatty acid oxidation. In Cellular Compartmentalization and Control of Fatty Acid Metabolism, pp. 39-63. Edited by C. Gran. Academic Press: New York.

Goldstein, L., and Newsholme, E. A. (1976). The formation of alanine from amino acids in diaphragm muscle of the rat. Biochemical Journal, 154, 555-558.

Gulotta, F., Payk, T. R., and Solbach, A. (1974). Sudanophile (mitochondriale) myopathie. Zeitschrift fur Neurologie (Berlin), 206, 309-326.

Jerusalem, F., Engel, A. G., and Peterson, H. A. (1975). Human muscle fiber fine structure: Morphometric data on controls. Neurology (Minneapolis), 25, 127-134.

Jerusalem, F., Spiess, H., and Baumgartner, G. (1975). Lipid storage myopathy with normal carnitine levels. Journal of the Neurological Sciences, 24, 273-282.

Karpati, G., Carpenter, S., Engel, A. G., Watters, G., 
Allen, J., Rothmann, S., Klassen, G., and Mamer, O. A. (1975). The syndrome of systemic carnitine deficiency. Clinical, morphological, biochemical, and pathophysiologic features. Neurology (Minneapolis), 25, 16-24.

Lehninger, A. L. (1970). Biochemistry, pp. 485-512. Worth Publishers: New York.

Lindstedt, G. (1967). Hydroxylation of gammabutyrobetaine to carnitine in rat liver. Biochemistry, 6, 1271-1282.

Markesbery, W. R., McQuillen, M. P., Procopis, P. G., Harrison, A. R., and Engel, A. G. (1974). Muscle carnitine deficiency. Association with lipid myopathy, vacuolar neuropathy and vacuolated leukocytes. Archives of Neurology (Chic.), 31, 320-324.

Marquis, N. R., and Fritz, I. B. (1964). Enzymological determination of free carnitine in rat tissues. Journal of Lipid Research, 5, 184-187.

Odessey, R. A., Khairallah, E. A., and Goldberg, A. L. (1974). Origin and possible significance of alanine production by skeletal muscle. Journal of Biological Chemistry, 249, 7623-7629.

Smyth, D. P. L., Lake, B. D., MacDermot, J., and Wilson, J. (1975). Inborn error of carnitine metabolism ("carnitine deficiency") in man. Lancet, 1, 1198-1199.

Sutton, E. H., and Wagner, R. P. (1975). Mutations and enzyme functions in humans. Annual Review of Genetics, 9, 187-212.

Vandyke, D. H., Griggs, R. C., Markesbery, W., and DiMauro, S. (1975). Hereditary carnitine deficiency of muscle. Neurology (Minneapolis), 25, 154-159. 